\title{
Influence of Annealed Temperature on Optical Properties of Nanostructured CdO Thin Films
}

\author{
Pradip Kumar Ghosh \\ Department of Physics, Abhedananda Mahavidyalaya, Sainthia, Birbhum, West Bengal, India
}

Email address:

pradipghosh2002@gmail.com,pkg_ju@yahoo.co.in

\section{To cite this article:}

Pradip Kumar Ghosh. Influence of Annealed Temperature on Optical Properties of Nanostructured CdO Thin Films. American Journal of Optics and Photonics. Vol. 5, No. 2, 2017, pp. 19-23. doi: 10.11648/j.ajop.20170502.12

Received: November 19, 2016; Accepted: January 12, 2017; Published: October 13, 2017

\begin{abstract}
Nanostructures of cadmium oxide (CdO) thin films were deposited by sol-gel dip coating technique on glass and $\mathrm{Si}$ substrates. X-ray diffraction patterns and selected area electron diffraction patterns confirmed the nanocrystalline cubic $\mathrm{CdO}$ phase formation. Transmission Electron Micrograph (TEM) of the film revealed the manifestation of nano CdO phase with average particle size lies in the range $1.6 \mathrm{~nm}$ to $9.3 \mathrm{~nm}$. From the measurements of transmittance spectra of the films the direct allowed bandgap values have been calculated and they lie in the range $2.85 \mathrm{eV}$ to $3.69 \mathrm{eV}$ with high transparency $(\sim 75 \%$ in the wavelength range $500-800 \mathrm{~nm}$ ) of the film. Particle size have also been calculated from the shift of bandgap from that of bulk value for those films for which the particles are compearable to Bohr exitonic radius.
\end{abstract}

Keywords: CdO Thin Films, Sol-gel, TEM, XRD, Optical Properties

\section{Introduction}

Nanocrystalline semiconductors have attracted much attention due to their novel properties and varaities of promising potentials in extensive applications [1, 2]. Numerous technical advancements in the field of nanostructured materials have stimulated the wide range of research interest in recent years because of various new properties exhibited by them. Recently, nanostructured semiconductor are widely used to design a rich varieties of device for microelectronics. One-dimensional nanostructured materials have gained special interest in the assembly of nanodevices [1-3]. Nanometer-scale electronics have been predicted to play an important role in device technology [4, 5]. Quantum wires of semiconductors [6] and metallic alloys [7] have found to exhibit interesting magnetic and electrical properties. The nanostructure transparent conducting oxides have also gained trememdous importance due to their size dependent optical properties and possible applications in near future.

Recently, various research groups around the world are working on the synthesis of several II-VI n-type transparent semiconducing oxide thin films in different process [8-18]. Previously, thin films of $\mathrm{CdO}$ have been synthesized by various techniques, including activated reactive evaporation
[8], spray pyrolysis $[9,10]$, solution growth [11], MOCVD [12], PLD [13], rf sputtering [14] etc. Recently, F doped CdO thin film have been reported by us [15] via sol-gel process. The preperation of $\mathrm{ZnO}$ quantum dots by Mahamuni et al [16], nanowires and nanoribbons byYao et. al [17], nanorods by Liu et. al [18] and Guo et. al [19] etchave been studied wiedly. The nanostructure of $\mathrm{CdO}$ have been prepared by Ashrafi et. al [20] via metalorganic molecular-beam epitaxy and nanobelts have also been prepared by Pan et. al [21] via thermal evaporation method. In this paper I report successful synthesis of nanostructured cadmium oxide thin film via a very simple solgel route. The sol-gel dip-coating method is chosen because of its many advantages such as easier composition control, better homogeneity, low processing temperature, lower cost, easier fabrication of large area films, possibility of using high purity starting materials and having an easy coating process of large and complex shaped substrates. In this paper, the temperature dependent of nanostructural and optical properties of cadmium oxide thin films has been studied.

\section{Experimental}

\subsection{Preparation of Films by Sol-gel}

The thin films of $\mathrm{CdO}$ have been deposited on glass and $\mathrm{Si}$ 
substrates using sol-gel dip coating process. Cadmium acetate $\left(99.99 \% \mathrm{Cd}\left(\mathrm{COOCH}_{3}\right)_{2}\right)$ has been taken as the source cadmium and 2-methoxy ethanol and monoethanol amine have been taken assolvent and stebilizer respectively. The solution of cadmium acetate dissolving in 2-methoxy ethanol was stirred by a magnetic stirrer and heated at a constant temperature of $\sim 80^{\circ} \mathrm{C}$ for a one hr. The solution was aged for three hrs. Before dip coating the glass substrates were cleaned by mild soap solution, washed thoroughly by distilled water and then in boiled water. Finally it was degreased in alcohol vapor. Si substrates were cleaned at first in $20 \%$ HF solution for 5 minutes and then washed in acetone in an ultrasonic cleaner. The cleaned substrates were dipped vertically into the solution and withdrawn very slowly at a speed of $\sim 8 \mathrm{~cm} / \mathrm{min}$ and dried at $60^{\circ} \mathrm{C}$ for 15 minutes for quick gel formation. This process was repeated for eight to nine times. Finally the coated substrates were annealed at desired constant temperature $200^{\circ} \mathrm{C}, 250^{\circ} \mathrm{C}, 300^{\circ} \mathrm{C}$ and $350^{\circ} \mathrm{C}$ for one $\mathrm{hr}$. in open air.

\subsection{Characterization}

The nanosructure and the selected area electron diffraction pattern of the films were studied by a transmission electron microscope (TEM, Hitachi-H600). X-ray diffraction pattern was recorded by an $\mathrm{X}$ - ray diffractometer (Bruker D8 Advance) in $2 \theta$ range $20-70^{\circ}$ using $\mathrm{Cu} \mathrm{K}_{\alpha}$ radiation of wavelength $\lambda=0.15406 \mathrm{~nm}$. The optical transmission spectra of the films were measured in the wavelength range $\lambda=300 \mathrm{~nm}$ to $800 \mathrm{~nm}$ using a UV-VIS-NIR spectrophotometer (Shimadzu UV-3101PC) at room temperature. The thickness of the film $(\sim 400 \mathrm{~nm})$ was estimated from cross-sectional scanning electron microscopy (SEM, JEOL-5200) measurement.

\section{Results and Discussion}

\subsection{Nanostructural Studies and X-ray Diffraction}

The nanostructured of the films, prepared by dispersing the nanoparticles on carbon coated copper grid, were studied at room temperature by using transmission electron microscope (TEM) which revealed the particle size lies in therange 1.6 $\mathrm{nm}$ to $9.3 \mathrm{~nm}$. The micrographs and corresponding diffraction pattern of $\mathrm{CdO}$ thin films for different annealing temperature have been shown in figure 1 (a) $200^{\circ} \mathrm{C}$, (b) $300^{\circ} \mathrm{C}$ and (c), SEAD for temperature $250^{\circ} \mathrm{C}$.

From SAED pattern, the inter-planer spacing (d) values have been calculated, which correspond to reflection from (111) and (220) planes of cubic CdO.

Table 1. Interplaner spacing (d) from TEM, XRD and JCPDS data card and corresponding (hkl) values.

\begin{tabular}{llll}
\hline $\mathbf{d}_{(\text {TEM) }}(\AA)$ & $\mathbf{d}_{\text {(XRD) }}(\AA)$ & $\mathbf{d}_{(\mathbf{J C P D S})}(\AA)$ & $(\mathbf{h k l )}$ \\
\hline 2.71 & 2.713 & 2.712 & $(111)$ \\
2.35 & 2.349 & 2.349 & $(200)$ \\
--- & 1.660 & 1.661 & $(220)$ \\
--- & 1.415 & 1.416 & $(311)$ \\
\hline
\end{tabular}

X-ray diffraction patterns of the $\mathrm{CdO}$ thin films using $\mathrm{Cu}$ $\mathrm{K}_{\alpha}$ radiation of wavelength $\lambda=1.5406 \AA$ has been shown in figure 2.

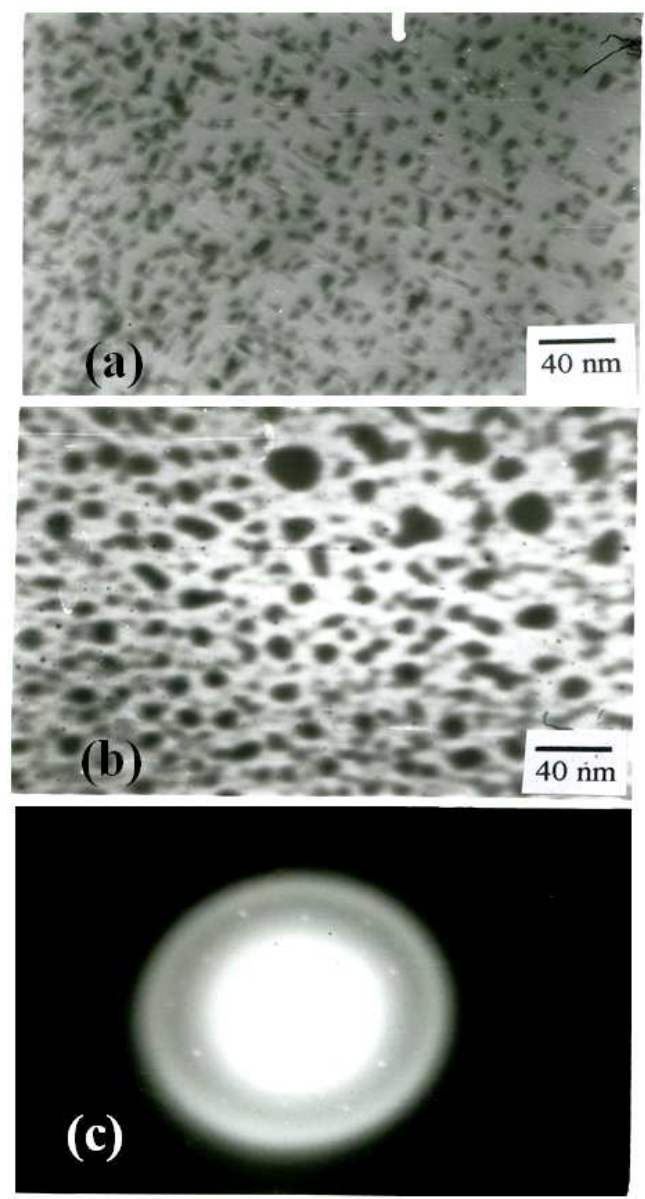

Figure 1. TEM micrographsand selected area electron diffraction pattern for (a) annealed temperature $200^{\circ} \mathrm{C}$, (b) annealed temperature $300^{\circ} \mathrm{Cand}$ (c) SAED pattern.

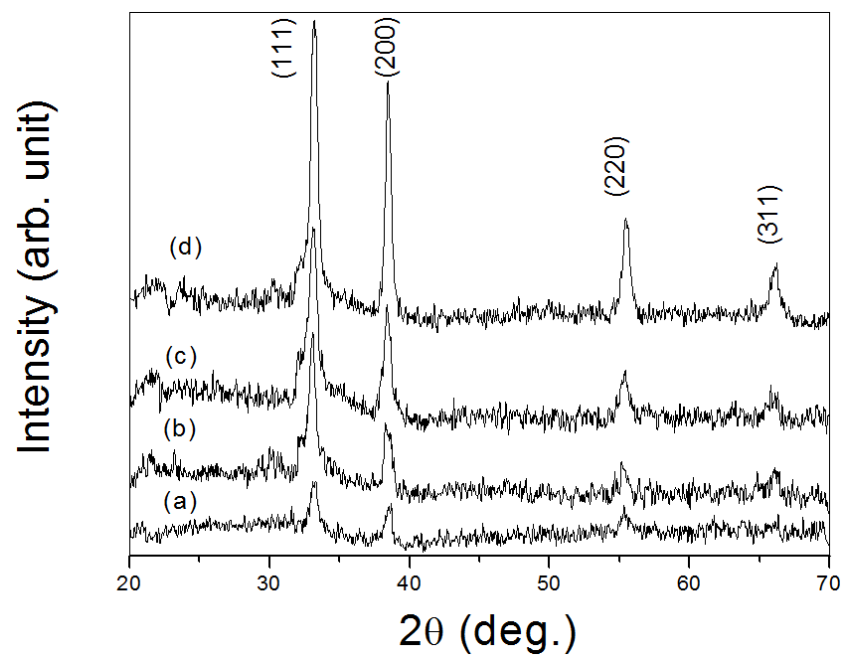

Figure 2. XRD paterns of a nanocrystalline $\mathrm{CdO}$ thin films deposited on glass substrates for annealed temperature (a) $200^{\circ} \mathrm{C}$, (b) $250^{\circ} \mathrm{C}$, (c) $300^{\circ} \mathrm{C}$ and (d) $350^{\circ} \mathrm{C}$. 


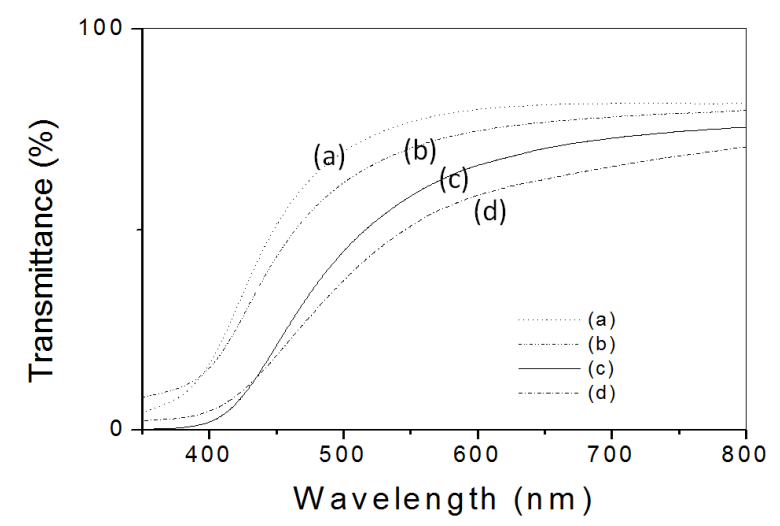

Figure 3. Transmittance spectra of representative nanocrystalline $\mathrm{CdO}$ thin films deposited on glass substrates at different annealed temperatures: (a) $200^{\circ} \mathrm{C}$, (b) $250^{\circ} \mathrm{C}$, (c) $300^{\circ} \mathrm{C}$ and (d) $350^{\circ} \mathrm{C}$.

The several peaks of cubic face-centered $\mathrm{CdO}$ with $\mathrm{a}_{0}=$ $4.6953 \AA$ have been obtained due to diffraction form (111), (200), (220) and (311) planes. The interplaner spacing (d) corresponding to XRD peaks, TEM measurement and JCPDS data card [22] have been compared as shown in table-1. Xray diffraction pattern of $\mathrm{CdO}$-nano shows broadening of peaks, which indicate $\mathrm{CdO}$ is nanocrystalline in nature.

\subsection{Optical Absorption and Optical Bandgap}

The optical bandgap values of the films $\mathrm{E}_{\mathrm{g}(\mathrm{film})}$ were determined from the transmission vs. wavelength traces. The absorption coefficient $(\alpha)$ was obtained from the standard relation [23]:

$$
\mathrm{T}=\mathrm{A} e^{-\frac{4 \pi k x}{\lambda}}
$$

where $\mathrm{k}$ is extinction coefficient, $k=\frac{\alpha \lambda}{4 \pi}$ and

$$
A=\frac{16 n_{0} n_{1}\left(n^{2}+k^{2}\right)}{\left\{\left(n_{0}+n\right)^{2}+k^{2}\right\}\left\{\left(n_{1}+n\right)^{2}+k^{2}\right\}}
$$

$\mathrm{n}_{0}, \mathrm{n}_{1}$ and $\mathrm{n}$ being the refractive index of air, substrate and films respectively.

For $\mathrm{k}^{2}<<\mathrm{n}^{2}$, $\mathrm{A}$ is nearly equal to unity and $\mathrm{T}$ can be expressed as:

$$
\mathrm{T} \sim \mathrm{e}^{-\alpha \mathrm{x}}
$$

The fundamental absorption, which corresponds to electron excitation from the valance band to conduction band, can be used to determine the nature and value of the optical band gap. The relation between the absorption coefficients $(\alpha)$ and the incident photon energy (hv) can be written as [24],

$$
(\alpha h v)^{1 / n}=A\left(h v-E_{g}\right)
$$

where $\mathrm{A}$ is a constant and $\mathrm{E}_{\mathrm{g}}$ is the bandgap of the material and exponent $\mathrm{n}$ depends on the type of transition. For direct allowed $n=\frac{1}{2}$, indirect allowed transition, $\mathrm{n}=2$, and for direct forbidden, $n=\frac{3}{2}$. To determine the possible transitions, $(\alpha h v)^{1 / n}$ vs. hv were plotted and corresponding bandgap were obtained from extrapolating the straight portion of the graph on hv axis at $\alpha=0$.

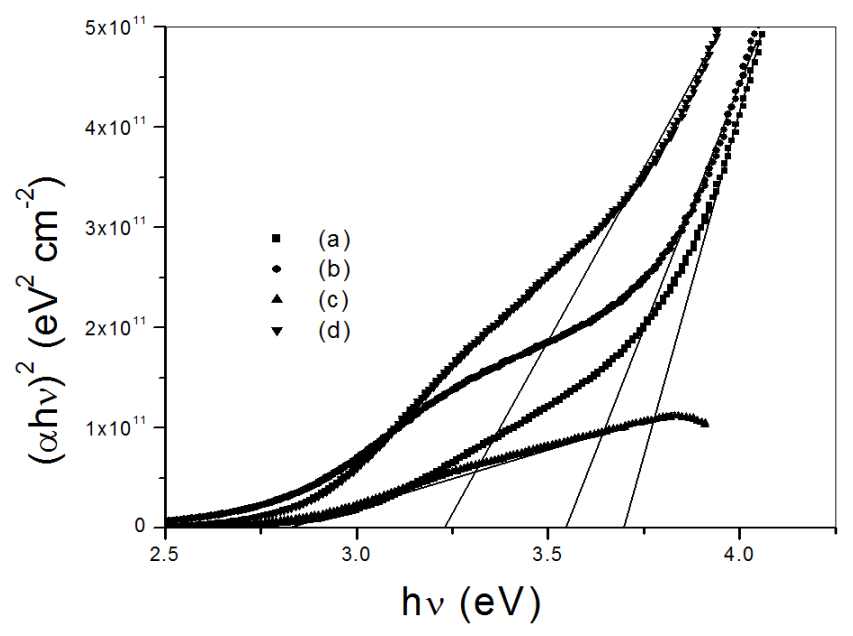

Figure 4. Determination of direct band gap of nanocrystalline CdO thin films for differentannealed temperatures: (a) $200^{\circ} \mathrm{C}$, (b) $250^{\circ} \mathrm{C}$, (c) $300^{\circ} \mathrm{C}$ and (d) $350^{\circ} \mathrm{C}$.

Figure 3 shows the transmttance vs. wavelength traces which show nearly $75 \%$ transmittance in the wavelength range of $500 \mathrm{~nm}$ to $800 \mathrm{~nm}$. To determine the possible transitions, $(\alpha h v)^{1 / n}$ vs. hv were plotted and corresponding band gap were obtained from extrapolating the straight portion of the graph on hv axis.

The direct bandgap calculated from $(\alpha h v)^{2}$ vs. hv plots (as shown in figure 4) lie in the range $2.85 \mathrm{eV}$ to $3.69 \mathrm{eV}$ and indirect bandgap calculated from $(\alpha h v)^{1 / 2}$ vs. hv plot lie in the range $1.6 \mathrm{eV}$ to $2.29 \mathrm{eV}$ as shown in figure 5. Both the direct bandgap and indirect bandgap values of the films are higher than that of the value of bulk materials because of quantum confinement of $\mathrm{CdO}$ nanocrystals.

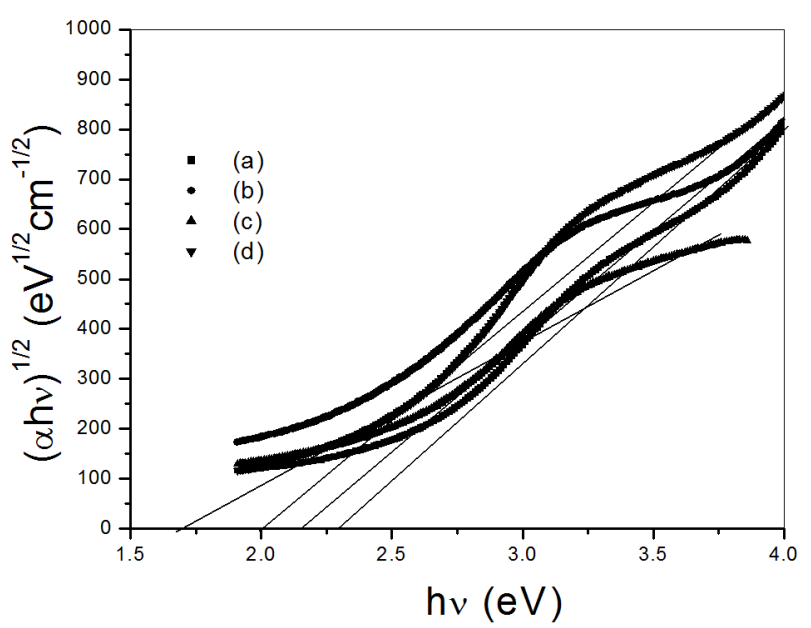

Figure 5. Determination of indirect band gap of nanocrystalline CdO thin films for different annealed temperatures: (a) $200^{\circ} \mathrm{C}$, (b) $250^{\circ} \mathrm{C}$, (c) $300^{\circ} \mathrm{C}$ and (d) $350^{\circ} \mathrm{C}$. 
The properties of nanocrystalline materials are changed from their corresponding bulk properties due to the sizes of the crystallites become comparable to the Bohr excitonic radius $\left(r_{B}\right)$.

$$
r_{B}=\frac{h^{2} \varepsilon}{\pi e^{2}}\left[\frac{1}{m_{e}{ }^{*}}+\frac{1}{m_{h}^{*}}\right]
$$

where $\varepsilon$ is the permittivity of the sample, $\mathrm{m}^{*}{ }_{\mathrm{e}}$ and $\mathrm{m}_{\mathrm{h}}{ }_{\mathrm{h}}$ are the effective mass of electron and hole in $\mathrm{CdO}$ respectively. The values of the particle size of the $\mathrm{CdO}$ thin films, as obtained from TEM studies for annealed temperature below $300^{\circ} \mathrm{C}$, are comparable to the Bohr excitonic radius supporting the quantum size effect.

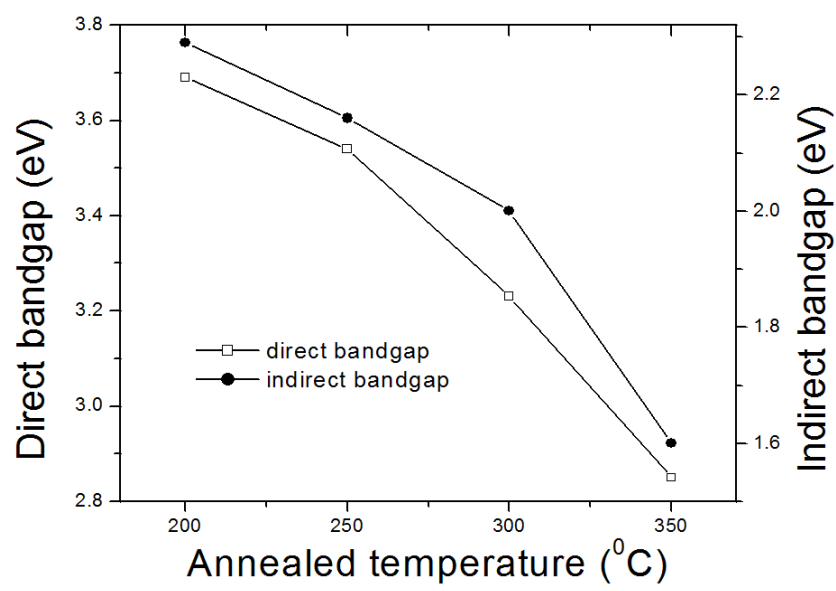

Figure 6. Variation of direct bandgap and indirect bandgap of nanocrystalline $\mathrm{CdO}$ thin film with annealed temperature.

The properties of nanocrystalline materials are changed from their corresponding bulk properties due to the sizes of the crystallites become comparable to the Bohr excitonic radius. The shift of band gap might also be utilized in determining the crystal radius (r) using relation $[25,26]$

$$
\Delta E_{g}=E_{g(\text { Film })}-E_{g(B u l k)}=\left[\frac{h^{2}}{8 \mu r^{2}}\right]-\left[\frac{1.8 e^{2}}{\varepsilon r}\right]
$$

where $\mu=\frac{1}{m_{e}{ }^{*}}+\frac{1}{m_{h}{ }^{*}}$ is the reduced mass of electronhole effective masses and $\varepsilon$ is the permittivity of the sample.

From the above equation, the particle sizes have been determined and these lie in the range $2.56 \mathrm{~nm}$ to $4.06 \mathrm{~nm}$ and that obtained from TEM measurements (bellow annealing temperature $300^{\circ} \mathrm{C}$ ) are also fairly support these results.

The variation of direct bandgap and indirect bandgap with different annealed temperature are shown in figure 6 . It is clear from this figure that both the direct bandgap and indirect bandgap decreases with increase in annealed temperature. These may due to better quantum confinement at comparatively lower annealing temperature.

\section{Conclusions}

The thin films of $\mathrm{CdO}$ nanoparticles have been successfully prepared by sol-gel process. XRD and SAED patterns confirmed the nanocrystalline cubic $\mathrm{CdO}$ phase formation. The XRD peaks are more broaden at lower temperature which indicate that the quantum confinement is better at lower temperature. TEM and optical studies also revealed that the quantum size effect occurred in the films. Optical transmission spectrum showed nearly $75 \%$ transmittance in the wavelength range of $500 \mathrm{~nm}$ to $800 \mathrm{~nm}$ and high direct bandgap lies in the range $2.85 \mathrm{eV}$ to $3.69 \mathrm{eV}$. The indirect bandgap of the films (lies in the range $1.6 \mathrm{eV}$ to $2.29 \mathrm{eV}$ ) are also higher than that of the bulk CdO materials. This may be due to quantum confinement effect of $\mathrm{CdO}$ nanoparticles.

\section{Acknowledgements}

The author wishes to thank Jadavpur University and Professor K. K. Chattopadhyay of Jadavpur University for providing him the opportunity of performing this work in Thin Films and Nanoscience Laboratory and inspiring him for this work.

\section{References}

[1] A. Bachtold, P. Hadley, T. Nakanishi and C. Dekker, Science 294, 1317 (2001).

[2] M. H. Huang, S. Mao, H. Feick, H. Q. Yan, Y. Y. Wu, H. Kind, E. Weber, R. Russo and P. D. Yang, Science 292, 1897 (2001).

[3] Y. Huang, X. F. Duan, Q. Q. Wei, and C. M. Lieber, Science 291, 851 (2001).

[4] R. F. Pease, in Nanostructures and mesoscopic systems, edited by W. P. Kirk and M. A. Reed (Academic, NewYork, 1992), p. 37.

[5] J. H. Schon, O. Schenker, B. Batlogg, Thin Solid Films, 385, 271 (2001).

[6] S. T. Lee, Y. F. Zhang, N. Wang, Y. H. Tang, I. Bello, C. S. Lee, and Y. W. Chung, J. Mater. Res. 14, 4503 (1993).

[7] H. J. Blythe, V. M. Fedosynk, O. I. Kasyutich, and W. Schwarzacher, J. Magn. Magn. Matter. 208, 251 (2000).

[8] G. Phatak, and R. Lal, Thin Solid Films 245, 17 (1994).

[9] K. Gurumurugan, D. Mangalaraj, and S. K. Narayandass, Thin Solid Films 251, 7 (1994).

[10] G. Sanatana, A. M. Acevedo, O. Vigil, F. Cruze, G. Contreraspuente, and L. Vaillant, Superficiesy Vacio 9, 300 (1999).

[11] A. Verkey, and A. F. Fort, Thin Solid Films 239,211 (1994).

[12] A. J. Freeman, K. R. Poeppelmeier, T. O. Mason, R.P.H. Chang, and T.J. Marks, MRS Bull. 25, 45 (2000).

[13] M. Yan, M. Lane, C. R. Kannewurf, and R. P. H. Chang, Appl. Phys. Lett. 78, 2342 (2001). 
[14] N. Ueda, H. Meada, H. Hosono, and H. Kawazoe, J. Appl. Phys. 84, 6174 (1998).

[15] P. K. Ghosh, R. Maity, K. K. Chattopadhyay, Sol. Energy Mat. \& Sol. Cells 81, 279 (2004).

[16] S. Mahamuni, K. Borgohain, B. S. Bendre, V. J. Leppert, S. H. Risbud, J. Appl. Phys. 852861 (1999).

[17] B. D. Yao, Y. F. Chan, N. Wang, Appl. Phys. Lett., 81, 757 (2002).

[18] B. Liu, H. C. Zeng, J. Am. Chem. Soc. 1254430 (2003).

[19] L. Guo, Y. L. Ji, H. Xu, P. Simon, Z. Wu, J. Am. Chem. Soc. 124, 14864 (2002)
[20] A. B. M. A. Ashrafi, H. Kumano, I. Suemune, Y. W. Ok, T. Y. Seong, J. Crys. Growth, 237-239, 518 (2002).

[21] Z. W. Pan, Z. R. Dai, Z. L. Wang, Science, 291, 1947 (2001).

[22] JCPDS Powder Diffraction file card5 - 0640.

[23] J. C. Manifacier, M. Demurcia, J. P. Fillard and E. Vicario, Thin Solid Films 41127 (1977).

[24] Optical Processes in Semiconductors, Pankove, Prentice-Hall. Inc., (1971).

[25] Y. S. Yuang, F. Y. Chen, Y. Y. Lee and C. L. Liu, Jpn. J. Appl. Phys. 76, 3041 (1994).

[26] A. D. Yoffe, Adv. In Phys. 42, 173 (1993). 